\title{
Directional invisibility by genetic optimization
}

\author{
Emre Bor, ${ }^{1,2}$ Ceren Babayigit, ${ }^{1}$ Hamza Kurt, ${ }^{1}$ Kestutis Staliunas, ${ }^{3,4}$ and Mirbek Turduev ${ }^{2, *}$ \\ ${ }^{1}$ Department of Electrical and Electronics Engineering, TOBB University of Economics and Technology, Ankara 06560, Turkey \\ ${ }^{2}$ Department of Electrical and Electronics Engineering, TED University, Ankara 06420, Turkey \\ ${ }^{3}$ Departament de Física, Universitat Politècnica de Catalunya (UPC), Colom 11, E-08222 Terrassa, Barcelona, Spain \\ ${ }^{4}$ Institució Catalana de Recerca i Estudis Avançats (ICREA), Passeig Lluís Companys 23, E-08010, Barcelona, Spain \\ *Corresponding author: mirbek.turduev@tedu.edu.tr
}

Received 13 August 2018; revised 26 October 2018; accepted 31 October 2018; posted 1 November 2018 (Doc. ID 342235); published 26 November 2018

In this Letter, the design of a directional optical cloaking by a genetic algorithm is proposed and realized experimentally. A three-dimensional finite-difference time-domain method is combined with the genetic optimization approach to generate the cloaking structure to directionally cloak a cylindrical object made of a perfect electrical conductor by suppressing the undesired scattered fields around the object. The optimization algorithm designs the permittivity distribution of the dielectric polylactide material to achieve an optical cloaking effect. Experimental verifications of the designed cloaking structure are performed at microwave frequencies, where the proposed structure is fabricated by $3 \mathrm{D}$ printing. The imperfect conformal mapping from a large-scale permittivity distribution and the compensation of the remaining scattering by a small-scale permittivity distribution are the basic physical mechanisms of the proposed optical cloaking. @ 2018 Optical Society of America

https://doi.org/10.1364/OL.43.005781

One of the most fascinating optical illusions is invisibility/ cloaking, when a space region or an object is concealed from an incident wave for a particular wavelength range. In early studies, conformal mapping approach was proposed to achieve invisibility of an object where the transformation optics (TO) was applied to design the cloaking $[1,2]$. Theoretical foundations to generate invisibility using $\mathrm{TO}$ were laid a decade ago, which reveal that host materials with anisotropic, negative, spatially varying permittivity, and permeability are required - the materials that do not exist in nature [3]. Furthermore, TO cloaks are narrowband and inherently lossy, which make them unattractive in the optical spectrum range. Hence, alternative solutions on the invisibility phenomenon are desirable.

The carpet cloaking is an alternative approach to hide objects under a special refractive layer $[4,5]$. Another approach to eliminate the scattering response of an object by using the Hilbert transform, and modifications of the Hilbert transform, was proposed [6-8]. Additionally, metamaterials are utilized to design an optical cloak based on coordinate transformation [9]. Furthermore, axisymmetric photonic crystal structures were introduced for approximate optical cloaking [10]. One more approach based on optical neutrality of metamaterials provides the invisibility without invoking cloaking [11]. Recently, the idea of using topology optimization algorithm also has become a promising approach to design cloaking structures [12-18].

In this Letter, we alternatively propose the application of a genetic algorithm (GA) to design a cloaking structure to conceal an object, in particular, a perfect electric conductor (PEC) material, from an incident plane wave propagating in a given direction and of a particular frequency. The designed cloaking structure is composed of polylactide (PLA) material which is a low-loss biodegradable thermoplastic polymer with low permittivity value. This dielectric material is widely used in 3D printing technology [14,19]. The physical mechanism of the directional concealing effect is primarily associated with the imperfect conformal mapping due to large-scale variation of the permittivity distribution around the object. This distribution, which is designed by a GA, provides a partial cloaking of the arbitrary object. However, as the cloaking by realistic materials cannot be perfect (the perfect cloaking requires negative permittivities and unrealistic permeabilities), the remaining scattering is present. The remaining scattering is eliminated by the small-scale modulation of the permittivity around the object, also rendered by the GA.

In this Letter, to achieve the desired manipulation of the propagating light, we optimized the permittivity distribution $\varepsilon(x, y)$ of the host medium, i.e., GA is used for the optimal distribution of permittivity to directionally cloak the PEC object. The GA is an evolutionary algorithm based on the survival of the fittest in the evolutionary process. As the biological counterparts of the evolution theory, the GA composes the mechanisms of crossover, mutation, and selection, and iteratively searches the solution space to find optimum solutions by minimizing a cost function [20].

The design for directional optical cloaking is schematically represented in Fig. 1(a). An incident plane wave with transverse-electric polarization propagates along the $x$-direction in free space, where the electric field components lie in the $x y$ - plane $\left(E_{x}, E_{y}\right)$, and the magnetic field $\left(H_{z}\right)$ is perpendicular to that plane. If the incident wave encounters an object, strong scattering is inevitable. Hence, to conceal the PEC object from the incident wave by diminishing the scattering effect, one 

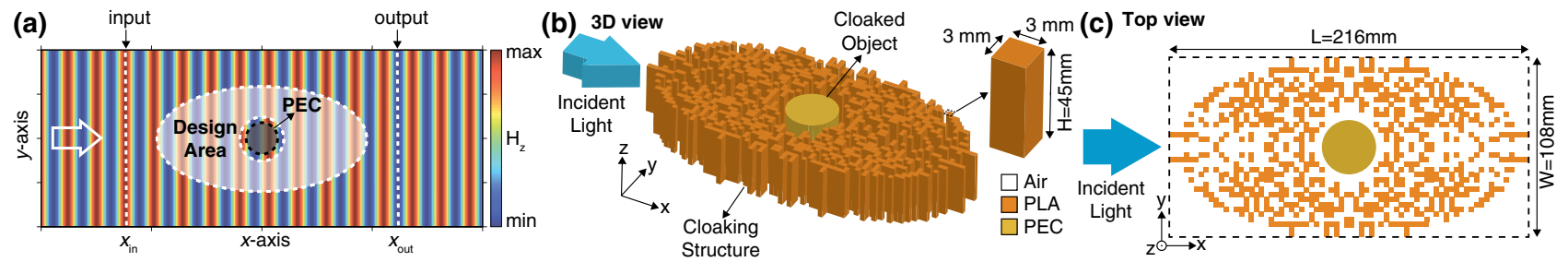

Fig. 1. (a) Schematic representation of the design approach. (b) Three-dimensional and (c) top views of the designed cloaking structure. The arrows indicate the direction of propagation for the incident plane wave.

should design a coating medium that surrounds the scattering object and ensures the undistorted plane wave behind the object.

We propose an all-dielectric ellipsoidal enveloping area, as shown in Fig. 1(a), which consists of square individual cells. The 3D finite-difference time-domain (FDTD) method is applied with the GA to numerically analyze the response of the designed cloaking structure during the optimization process [21]. The employed GA operates with binary numbers, i.e., the individual cells made of PLA material are considered as "1" and air holes/cells are considered as " 0 " within the optimization region. The sizes of the corresponding individual cells are $3 \mathrm{~mm} \times 3 \mathrm{~mm}$ with heights of $45 \mathrm{~mm}$, where the material permittivity of PLA dielectric and air are $\varepsilon_{\text {PLA }}=2.4025$ and $\varepsilon_{\text {air }}=1.0$, respectively.

Since we deal with directional concealing, we first consider the field scattered in front of and behind the design area, the so called input and output planes. For this reason, we examine the cross sections of the field distributions along the transverse $y$ - direction at input and output planes denoted by the dashed lines in Fig. 1(a). The cloaked structure is illuminated by a plane wave source at a frequency of $10 \mathrm{GHz}$. During the optimization process, the profiles of scattered fields at input and output cross-sectional planes are corrected, i.e., reshaped to form the plane waves. We minimize the deviation of the cross-sectional field profiles from the average values. For this purpose, the primary objective of directional concealing is to minimize the error between the oscillation of the field profiles and average values that can be expressed as follows:

$$
\begin{aligned}
H_{\text {error }}= & \sum_{y}\left(\left|H_{z}\left(x_{\text {in }}, y\right)-\bar{H}_{z}\left(x_{\text {in }}, y\right)\right|\right. \\
& \left.+\left|H_{z}\left(x_{\text {out }}, y\right)-\bar{H}_{z}\left(x_{\text {out }}, y\right)\right|\right),
\end{aligned}
$$

where the overbars stand for the average values of the crosssectional profiles. In Eq. (1), $H_{z}$ represents the cross-sectional magnetic field profile, and $y$ denotes the order of the mesh cells in the simulation area along the $y$-axis. The terms $x_{\text {in }}$ and $x_{\text {out }}$ denote [see Fig. 1(a)] the positions of the profiles for the input and output planes in the $x$-direction, respectively. The input and output locations are selected to be $30 \mathrm{~mm}$ away from the edge of the cloaking structure in the $x$-direction. The algorithm also aims to reduce the distortion of the phase fronts at the input and output positions. The corresponding phase front error can be expressed as follows:

$$
\begin{aligned}
\varphi_{\text {error }}= & \sum_{y}\left(\left|\varphi_{z}\left(x_{\text {in }}, y\right)-\bar{\varphi}_{z}\left(x_{\text {in }}, y\right)\right|\right. \\
& \left.+\left|\varphi_{z}\left(x_{\text {out }}, y\right)-\bar{\varphi}_{z}\left(x_{\text {out }}, y\right)\right|\right),
\end{aligned}
$$

where $\varphi_{z}$ represents the cross-sectional phase profile. The design of a cloaking structure is a multi-objective error minimization problem; thus, the algorithm minimizes the sum of error values $H_{\text {error }}$ and $\varphi_{\text {error }}$. Moreover, the transmission efficiency of the initially designed cloaking structure should be maximized during the optimization process, since proper optical concealing of an object will be reasonable with high transmission efficiency. For this reason, we subtract the calculated transmission efficiency $(T)$ from the sum of the error values resulting in the following cost function:

$$
f_{\text {cost }}=H_{\text {error }}+\varphi_{\text {error }}-T .
$$

The $3 \mathrm{D}$ view and top view of the designed cloaking structure are given in Figs. 1(b) and 1(c), respectively. Here the optimized cloaking structure consists of dielectric and air individual cells genuinely located in the design area to cloak a PEC cylindrical object with a diameter of $36.4 \mathrm{~mm}$ and height of $45 \mathrm{~mm}$. The total width and length of the designed cloaking structure are calculated as $W=108 \mathrm{~mm}$ and $L=216 \mathrm{~mm}$, respectively. As can be seen from Fig. 1(b), the resulting cloak design is a binary structure. Efficient light manipulation ability and simplicity of this type of binary structure have already been discussed in the literature [22].

In order to examine the time-domain response of the optimized cloaking structure, comparative plots of a cylindrical PEC object with and without a cloaking structure exposed to the incident plane wave are presented in Fig. 2. The magnetic field distributions and corresponding phase profiles are shown in Figs. 2(a) and 2(b), respectively, for the cases of free-space wave propagation, field scatterings due to the PEC object, the scattering field correction by the optimized cloaking structure, and the designed structure without a PEC object. Here the magnetic field and phase distributions are extracted at the $z=0$ cross-sectional $x y$ - plane in the $3 \mathrm{D}$ FDTD simulation region. The calculated profiles at the input and output planes are given in Fig. 2(c). As can be seen, the PEC object strongly scatters the incident plane wave and leads to strong variations in both the magnetic field and phase distributions. The designed cloaking structure around the PEC object suppresses the field variations and successfully reproduces the incident plane wave at the back cross section. The deformation of the magnetic field distribution at the input area, however, is not completely eliminated. The calculated transmission efficiencies for the cases of free space, PEC, and PEC with the cloaking structure and the cloaking structure without PEC are superimposed in Fig. 2(d). It can be deduced that the cloaking structure is optimized in such a way that undesired reflections from the cloaking region are negligibly small, i.e., the cloaking region acts as a transparent/anti-reflective coating effect that bends the light around the PEC object. 

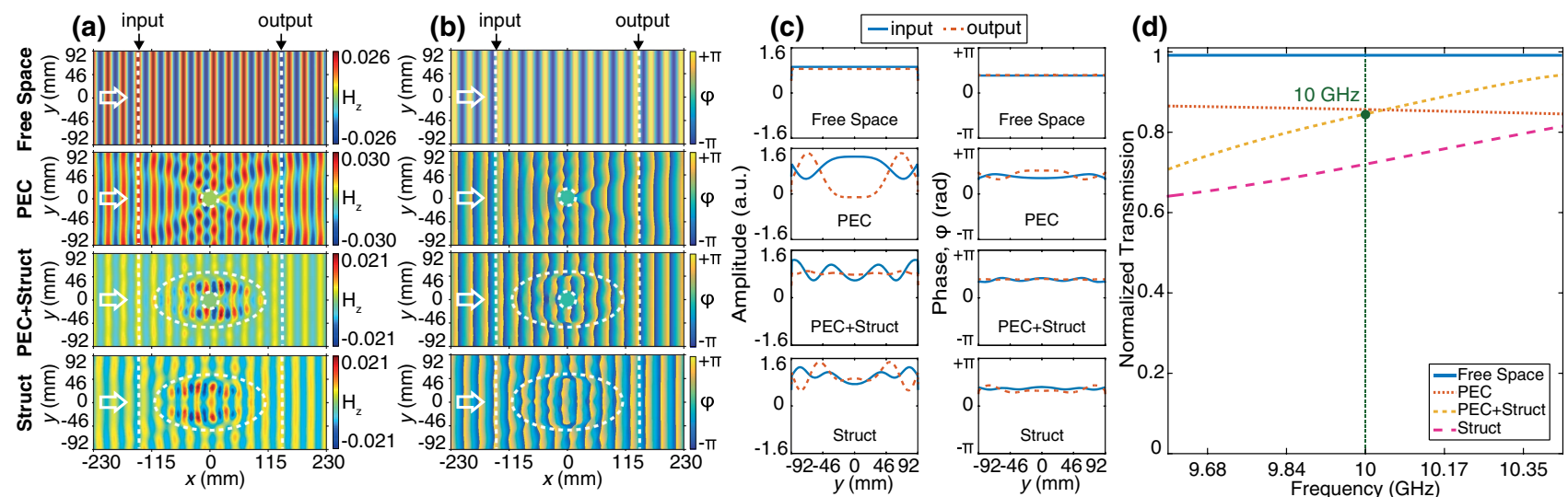

Fig. 2. Calculated (a) magnetic field and (b) phase distributions with (c) their cross-sectional amplitude and phase profiles at the front and back cross sections are given for free-space propagation, the PEC object, the cloaked PEC object, and the cloaking structure without the PEC object, respectively, from top to bottom. The performance of only the cloaking structure (without the cloaked object) is given for comparison in the bottom row. The arrows indicate the propagation direction of the incident wave. The position profiles at the input and output of the cross sections are denoted by the dashed lines. The dashed circles and dashed ellipsoids represent the boundaries of the PEC material and of the designed cloaking structure, respectively. (d) Transmission efficiencies of three different cases are plotted, where the green dashed vertical lines indicate the selected design frequency of $10 \mathrm{GHz}$.

In fact, wave propagation through the cloaked region can give key information for the physical mechanism of the concealing effect. An observed, the optical cloaking effect can be explained as a combination of two different physical mechanisms. First, the designed cloaking structure around the cylindrical PEC object provides a partial cloaking via TO and conformal mapping due to the mean field effects of the discretized structure $[1,2]$. Inspections of the steady-state magnetic field distribution in Fig. 2(a) indicate that the incident plane wave splits into two branches and bends around the PEC object inside the cloaking structure. Moreover, the bended light outside the cloaking region is weakly resonating around the PEC object. Note that the optical path length is increased within the cloaking structure which can be considered as an inhomogeneous medium for the incident plane wave. On the other hand, the waves propagating through and around the cloaking structure match well to maintain the plane wave propagation of the incident light behind the cloaking structure. Hence, the incident plane wave bends and propagates around the object which resembles the cloaking via conformal mapping.

The full conformal mapping requires unrealistic values of electric permittivity and magnetic permeability to provide the $100 \%$ cloaking $[1,2]$. Since we are restricted to the realistic values of the permittivities, the designed structure never provides $100 \%$ cloaking of an object. This is justified by the bottom row in Fig. 2 when the cloaked object is removed; some fraction of the field penetrates through the cloak to the position of the removed object. This is what we mean under "imperfect cloaking." As a result, there remains a weak rudimental scattering from the object due to the imperfect cloaking which depends on the particular form of the object. The second mechanism is the elimination of that weak scattering by creating an inhomogeneous medium for light to travel in a tailored optical path. The combination of these two mechanisms of the cloaking result in efficient cloaking that is achieved by realistic values of electric permittivity and magnetic permeability.

The experimental verification of the cloaking effect of the optimized structure was performed at a microwave regime, using the $3 \mathrm{D}$ printed cloaking structure from the PLA material. The permittivity of the $3 \mathrm{D}$ printed PLA material was $\varepsilon_{\text {PLA }}=2.4025$, according to the Nicolson-Ross and Weir measurement method [23]. Since PEC is an ideal material with infinite electrical conductivity which does not exist in nature, we use a cylindrical brass object to be cloaked. Brass (mix of copper and zinc) is a metallic alloy, which strongly scatters the incident wave at microwave frequencies at around $10 \mathrm{GHz}$. The visual representation of the microwave experimental setup is shown in Fig. 3(a). The photographic view of the fabricated cloaking structure is given in Fig. 3(b) with a cylindrical brass object inside. For the experiments, an Agilent E5071C ENA vector network analyzer was used as a microwave generator. The generated microwaves at an operating frequency of $10 \mathrm{GHz}$ were directed towards the cloaking structure by the horn antenna in front of the structure. As the horn antenna generates a Gaussian profiled wave with spherical wavefronts, it is positioned at a sufficiently large distance from the cloaking region to have the input close to the plane wave. A monopole antenna is also connected to the same network analyzer and placed on a motorized stage in order to measure the magnetic field distribution with its phase profile behind the designed cloaking structure. Here the measurement of the magnetic field $\left(H_{z}\right)$ and phase $\left(\phi_{z}\right)$ components at the scanning area is realized by sweeping the monopole antenna with $2 \mathrm{~mm}$ steps along both the $x$-axis and $y$-axis. In the experiments, initially, we placed the brass object, and then scanned the area behind it to demonstrate the deterioration of the propagating wave. Next, we inserted the brass object into the center of the cloaking structure; then we measured the magnetic field and phase components behind the cloaking structure. The corresponding magnetic fields and phase distributions for the measurements with and without a cloaking structure are shown in Figs. 3(c) and 3(d), respectively. As can be seen from Fig. 3(c), a cylindrical brass object highly scatters the incident wave, i.e., it is divided into two branches. On the other hand, the cloaking structure collects the scattered fields and canalizes them around the object to form a plane wave in 

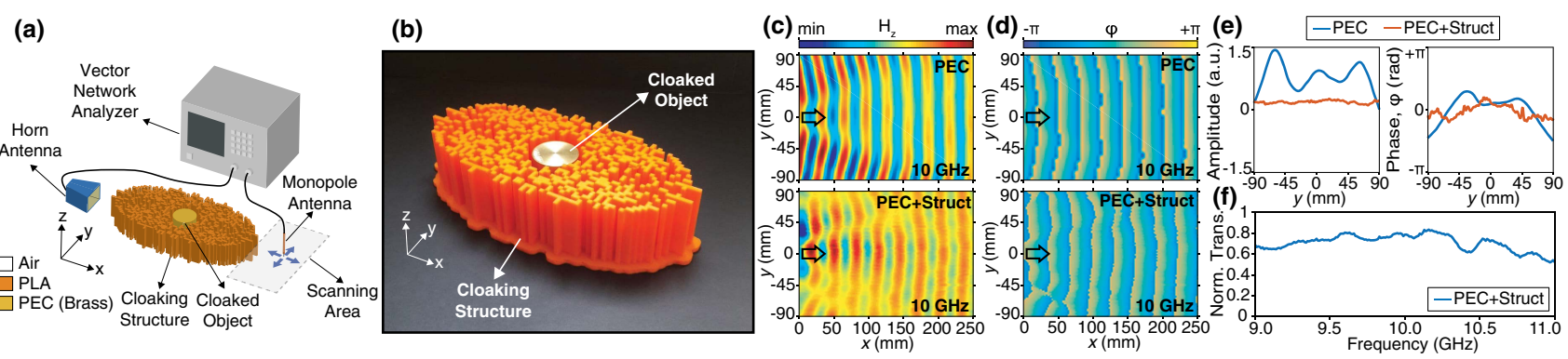

Fig. 3. (a) Schematic representation of the experimental setup and (b) a photo of the fabricated cloaking structure with a cylindrical brass object which was used as the PEC object. (c) Magnetic field and (d) phase distributions of scatterings due to the PEC material (top) and the correction of the plane wave by the cloaking structure (bottom) at the output plane. (e) Experimentally measured cross-sectional amplitude and phase profiles. (f) Measured transmission efficiency of the cloaking structure with the PEC (brass) object placed at the middle of the structure.

the scanning area. According to the given phase distributions in Fig. 3(d) (bottom), the planar phase fronts indicate that the cloaking structure was able to reproduce the incident plane wave. In addition, a cross-sectional magnetic field and phase profiles are plotted in Fig. 3(e). In addition to the field and phase profile results, the transmission efficiency of the cloaking structure was measured and represented in Fig. 3(f). The measured transmission efficiency is around $75 \%$ at $10 \mathrm{GHz}$, and the result is very close to the numerical value.

Although the main purpose of the Letter was the demonstration of the novel approach of cloaking at a fixed frequency, some comments on the cloaking bandwidth are also in order. Both approaches discussed that the physical mechanisms of cloaking can be considered as the impedance matching by the cloaking structure. The frequency bandwidths of passive cloaking structures are generally restricted by the Bode-Fano condition [24], with some modifications for the active cases [25]. Therefore, the universal restrictions by the Bode-Fano condition can also be applied for our case. However, the detailed analysis of the cloaking bandwidth was outside the scope of the Letter. A possible subsequent study, in principle, could employ our genetic optimization procedure using a frequency-dependent target function in order to obtain the maximum bandwidth (within the Bode-Fano bounds).

Concluding, we proposed the design of a cloaking structure by utilizing the GA integrated with a 3D FDTD method. The numerically calculated magnetic field and phase distributions imply that the designed cloaking structure is able to suppress the scattered fields due to an object in order to reproduce the incident plane wave at the input and output with high transmission efficiency. The proposed cloaking structure was fabricated by a $3 \mathrm{D}$ printing technique, and a cylindrical brass object is selected as a highly scatterer to be cloaked at microwave frequencies. Thus, the working principle of optical cloaking is also experimentally demonstrated at microwave frequencies at around $10 \mathrm{GHz}$. A good agreement between the numerical and experimental results is achieved. The physical mechanisms of cloaking are related with partially realized conformal mapping and intelligent distribution of permittivity on a small scale to reduce the weak scatterings from the cloaked object. In conclusion, the proposed design approach is not restricted to directional optical cloaking, but also can be applied for different cloaking scenarios dealing with electromagnetic and acoustic waves.
Funding. Türkiye Bilimsel ve Teknolojik Araştirma Kurumu (TÜBITAK) (116F182); NATO SPS (985048); Spanish Ministerio de Ciencia e Innovación; European Union FEDER (FIS2015-65998-C2-1-P).

Acknowledgment. K.Staliunas acknowledges the support of the NATO SPS Research and the Spanish Ministerio de Ciencia e Innovación and European Union FEDER. H. Kurt acknowledges the partial support of the Turkish Academy of Sciences.

\section{REFERENCES}

1. U. Leonhardt, Science 312, 1777 (2006).

2. J. B. Pendry, D. Schurig, and D. R. Smith, Science 312, 1780 (2006).

3. H. Chen, C. T. Chan, and P. Sheng, Nat. Mater. 9, 387 (2010).

4. J. Li and J. B. Pendry, Phys. Rev. Lett. 101, 203901 (2008).

5. J. Valentine, J. Li, T. Zentrgraf, G. Bartal, and X. Zhang, Nat. Mater. 8, 568 (2009).

6. S. A. R. Horsley, M. Artoni, and G. C. La Rocca, Nat. Photonics 9, 436 (2015).

7. Z. Hayran, R. Herrero, M. Botey, H. Kurt, and K. Staliunas, Phys. Rev. A 98, 013822 (2018).

8. Z. Hayran, R. Herrero, M. Botey, H. Kurt, and K. Staliunas, ACS Photonics 5, 2068 (2018).

9. W. Cai, U. K. Chettiar, A. V. Kildishev, and V. M. Shalaev, Nat. Photonics 1, 224 (2007).

10. D. Xiao and H. T. Johnson, Opt. Lett. 33, 860 (2008).

11. R. Hodges, C. Dean, and M. Durach, Opt. Lett. 42, 691 (2017).

12. J. Andkajaer and O. Sigmund, Appl. Phys. Lett. 98, 021112 (2011).

13. L. Lan, F. Sun, Y. Liu, C. Ong, and Y. Ma, Appl. Phys. Lett. 103 121113 (2013).

14. B. Vial, M. M. Torrico, and Y. Hao, Sci. Rep. 7, 3929 (2017).

15. J. Andkjaer, N. A. Mortensen, and O. Sigmund, Appl. Phys. Lett. 100, 101106 (2012).

16. G. Fujii, H. Watanabe, T. Yamada, T. Ueta, and M. Mizuno, Appl. Phys. Lett. 102, 251106 (2013).

17. Y. Urzhumov, N. Landy, T. Driscoll, D. Basov, and D. R. Smith, Opt. Lett. 38, 1606 (2013).

18. B. Vial and Y. Hao, Opt. Express 23, 23551 (2015).

19. B. M. Boyle, T. A. French, R. M. Pearson, B. G. McCarthy, and G. M. Miyake, ACS Nano 11, 3052 (2017).

20. M. Melanie, An Introduction to Genetic Algorithms (MIT Press, 1999).

21. Lumerical Inc., http://www.lumerical.com/tcad-products/fdtd/.

22. C. D. Giovampaola and N. Engheta, Nat. Mater. 13, 1115 (2014).

23. W. B. Weir, Proc. IEEE 62, 33 (1974).

24. F. Monticone and A. Alù, Optica 3, 718 (2016).

25. P.-Y. Chen, C. Argyropoulos, and A. Alù, Phys. Rev. Lett. 111, 233001 (2013). 